\title{
Comparison of Nested PCR and Conventional Analysis of Plasmodium Parasites in Kano, Nigeria
}

\author{
Oladele Olasoji Vincent ${ }^{1,2}$, Eugene Ikeh ${ }^{1}$, Feyi Obisakin ${ }^{3}$ \\ ${ }^{1}$ Department of Medical Microbiology, Faculty of Medical Sciences, University of Jos, Plateau, Nigeria \\ ${ }^{2}$ Department of Educational Services, Universal Basic Education, Abuja, Nigeria \\ ${ }^{3}$ National Veterinary Research Institute, Jos, Plateau, Nigeria \\ Email address: \\ Olasoji2002@gmail.com (O. O. Vincent)
}

\section{To cite this article:}

Oladele Olasoji Vincent, Eugene Ikeh, Feyi Obisakin. Comparison of Nested PCR and Conventional Analysis of Plasmodium Parasites in Kano, Nigeria. European Journal of Clinical and Biomedical Sciences. Vol. 3, No. 5, 2017, pp. 85-90. doi: 10.11648/j.ejcbs.20170305.11

Received: August 10, 2017; Accepted: September 5, 2017; Published: October 5, 2017

\begin{abstract}
Plasmodium identification represents the crucial factor in malaria diagnosis and treatment across developing countries. Conventional microscopy and the use of rapid diagnostic kits have been extensively applied towards human malaria diagnosis. Recombinant DNA techniques have been applied towards malaria diagnosis as well as in the species specific identification using Plasmodium 18s-rRNA gene. This study was undertaken amongst patients attending the Murtala Mohammed Specialist Hospital, Kano. Blood samples were collected from 350 malaria suspected patients. Microscopic analysis via Giemsa-staining revealed that 220 patients were positive for malaria. RDT analysis showed that 248 test samples were positive for Plasmodium infection. DNA products obtained from the blood samples were analyzed by nested PCR to amplify the 18S ssrRNA Plasmodium gene with genus and specific primers rPLU1/5, rPLU3/4, rVIV1/2, rFAL1/2, rMAL1/2 and rOVA1/2. Data obtained showed that $58.64 \%$ of specimens tested by microscopy were false positives while $60.62 \%$ of false positives were obtained using RDTs in comparison to nPCR which proved that on 91 out of 350 patients were infected with Plasmodium falciparum, representing $26 \%$ of tested specimen. Comparative analysis of nPCR to microscopy showed that the sensitivity and positive predictive values of the nPCR were determined as 100 and $41.36 \%$, respectively, while against RDTs it was 100 and $39.38 \%$ respectively. nPCR was determined to be more sensitive and specific than either microscopy or RDTs. This study revealed that the accurate diagnosis of malaria by nPCR was compulsory in malaria-prone regions of Nigeria such that nPCR should be applied routinely in laboratory studies.
\end{abstract}

Keywords: Microscopy, RDTs, Nested PCR, Plasmodium falciparum, Kano

\section{Introduction}

Malaria is a major disease, emanating from parasites of the Plasmodium genus, which plagues mankind with over 200 million cases reported annually [1]. Reports indicate that the scourge of malaria claims the lives of between 438,000 to 655,000 patients per year of which over $80 \%$ of the victims are children between 1-5 years of age, the majority of which cuts across sub-Sahara Africa [1-3]. Data revealed that in Nigeria, the high occurrence of malaria, particularly in the northern region of the country is elicited by one of the four species of plasmodia; Plasmodium falciparum [4]. Other known members of this protozoan parasite family include Plasmodium malariae, Plasmodium ovale and Plasmodium vivax which collectively form cause malaria in humans of which $P$. falciparum is the cause of morbidity and mortality [5]. The number of cases still remains on the high side despite the availability of anti-malaria medication that has been commercially available for decades.

Strategies employed to avert the spread of malaria include the increased use of indoor residual spraying (IRS) canisters, application of insecticide treated nets (ITN), combination therapies using drug bases like artemisinin, phyto-medicines and nutraceutical approaches according to folklore as well as the use of rapid diagnostic test (RDT) have been embraced by affected regions but has not however eliminated the fatality rates associated with the disease [3], [6]. So far, early detection of asymptomatic infections presents the best possible approach to reduce and prevent the transmission of malaria which would otherwise go unnoticed [7]. Proper and 
rapid clinical identification of the species of Plasmodium is another crucial area in the fight against malaria. Studies show that in endemic regions, some affected individuals exhibit mixed infections which stem from two or more Plasmodium species and in some cases, these mixed infections; $P$. malariae and P. ovale, are overlooked [8-9].

Even with the use of RDTs, clinicians in developing countries still experience degrees of difficulty in the detection of mixed infections thus resulting in cases of ineffective treatment modalities that gives rise to mortality in severe cases [10]. The most common of the conventional methods used in the diagnosis of malaria is via light microscopic examination of thick or thin blood smears stained with Romanowsky stain. This procedure is however labour intensive and requires experts in clinical parasitology in addition to being time consuming. A quicker modern alternative involves the use to flow cytometric techniques or immuno-chromatographic approaches whereby detection is confirmed via antigen-antibody interaction [11-13]. In both approaches, clinicians are capable of detecting 50-100 parasites $/ \mu \mathrm{L}$ of infected blood sample. The success of the latter approach is however dependent on low levels of parasitemia and is specific for only the Plasmodium falciparum specie, negating the others [14]. In endemic regions where the pool of parasitemia is predominantly high and the possibility of mixed infections is high, these techniques would be ineffective towards the proper identification of the disease causing agent, thus leading to false-negative results [15]. As such, highly endemic areas like Nigeria require a simple, specie sensitive method for the early detection of malaria.

Recombinant DNA technologies has provided the ideal platform for the identification and distinction of Plasmodium species which in turn enhances diagnosis of even low-density parasitemia that are not picked up by conventional light microscopy [16-17]. The paradigm shift towards the use of molecular diagnostic approaches is catapulted by its ease of detecting multiple parasites per $\mu \mathrm{l}$ of blood irrespective of whether the sample contained single or multiple species through the use of gene specific primers for targeting [1821]. Despite such reports, not much has been covered in terms of genomic determination of Plasmodium species infecting patients who visit general and specialist hospitals in northern Nigeria. The aim of this study was then to access the possibility of using gene specific primers for Plasmodium towards malaria diagnosis from blood samples amongst patients in northern Nigeria.

\section{Materials and Methods}

\subsection{Study Area}

The study location was Murtala Mohammed Specialist hospital within Kano metropolis (latitudes $11^{\circ} 25^{\prime} \mathrm{N}$ to $12^{\circ} 47^{\prime}$ $\mathrm{N}$ and longitude $8^{\circ} 22^{\prime} \mathrm{E}$ to $\left.8^{\circ} 39^{\prime} \mathrm{E}\right)$, Nigeria. Kano metropolis is bordered by Madobi and Tofa Local Government Areas (LGAs) to the South West, Gezawa LGA to the East, Dawakin Kudu LGA to the South East, and Minjibir LGA on the North East.

\subsection{Ethical Permission}

Approval to conduct the study was obtained from the Kano State Ministry of Health Ethical Committee before sample collection from patients. Informed consent forms were also sought from willing patients and their guardians at the general outpatient department (GOPD) of Murtala specialist hospital Kano state, Nigeria.

\subsection{Blood Collection and Screening}

About $10 \mathrm{ml}$ of venous blood was collected from each study subject by venipuncture into Ethylenediamine tetra acetate (EDTA) tubes. Screening for malaria parasite was done using a rapid diagnostic kit; (SD BIOLINE Malaria Ag RDT) in accordance with the manufacturer's instruction. This kit is also used for the detection of specific antigen for the four Plasmodium species. $5 \mu$ l of blood samples were drawn using capillary pipettes and added into the round sample well touching the sample pad of the test kits. About 4 drops of assay diluent were added into the assay diluents well and allowed to stand for 12-15 minutes. The development of red band in both the control and test sample kits served as a positive test and absence of a red color on the test band signified a negative result.

\subsection{Molecular Analysis}

Template DNA was obtained from blood samples using the QIAamp Blood Kit (Cat. No.51106; Qiagen Inc., USA) according to the manufacturers' instruction. Species diagnostics was achieved through the amplification of the PCR (polymerase chain reaction) products of the 18S rRNA genes of Plasmodium species using the rPLU1 (5'TCAAAGATTAAGCCATGCAAGTGA-3') and rPLU5 (5'CCTGTTGTTGCCTTAAACTCC-3') primers for the first nested PCR [22]. In the first PCR, $2 \mu 1$ of template DNA (corresponding to approximately 0.25 to $0.5 \mu \mathrm{l}$ of blood) was added to a $20 \mu \mathrm{l}$ PCR mixture that consisted of $0.4 \mu \mathrm{M}$ each universal Plasmodium genus specific primer rPLU1 (forward primer) and rPLU5 (reverse primer), $200 \mu \mathrm{M}$ each deoxynucleoside triphosphate, $25 \mathrm{mM} \mathrm{MgCl}_{2}, 1$ X PCR Gold Buffer II (50 mM KCl, $15 \mathrm{mM}$ Tris-HCl, $\mathrm{pH} 8.0$ ), and $0.25 \mathrm{U}$ AmpliTaq Gold DNA polymerase. The PCR cycle ran at $94^{\circ} \mathrm{C}$ for $4 \mathrm{~min}$ and then 35 cycles at $94^{\circ} \mathrm{C}$ for $30 \mathrm{~s}, 55^{\circ} \mathrm{C}$ for $1 \mathrm{~min}$, and $72^{\circ} \mathrm{C}$ for $1 \mathrm{~min}$, followed by a final extension at $72^{\circ} \mathrm{C}$ for $5 \mathrm{~min}$. The first PCR product was diluted 20 -fold in sterile water.

$1 \mu \mathrm{L}$ of this solution was used for the second nested PCR cycle, performed at $94^{\circ} \mathrm{C}$ for $4 \mathrm{~min}$ and then 35 cycles at $94^{\circ} \mathrm{C}$ for $30 \mathrm{~s}, 58^{\circ} \mathrm{C}$ for $1 \mathrm{~min}, 72^{\circ} \mathrm{C}$ for $1 \mathrm{~min}$, followed by a final extension at $72^{\circ} \mathrm{C}$ for $5 \mathrm{~min}$ with the Plasmodium genus specific primer forward primer rPLU3 (5'TTTTTATAAGGATAACTACGGAAAAGCTGT-3') and the rPLU4 species-specific reverse primer (5'TACCCGTCATAGCCATGTTAGGCCAATACC-3'). 
Subsequent experiments used a second set of primers; rVIV1 (5'-CGCTTCTAGCTTAATCCACATAACTGATAC-3') and rVIV2 (5'-ACTTCCAAGCCGAAGCAAAGAAAGTCCTTA3') for $P$. vivax, rFAL1 (5'TTAAACTGGTTTGGGAAAACCAAATATATT-3') and rFAL2 (5'-ACACAATGAACTCAATCATGACTACCCGTC3') for $P$. falciparum, rMAL1 (5'ATAACATAGTTGTACGTTAAGAATAACCGC-3') and rMAL2 (5' - AAAATTCCCATGCATAAAAAATTATACAAA3') for $P$. malariae, rOVA1 (5'ATCTCTTTTGCTATTTTTTAGTATTGGAGA-3') and rOVA2 (5'-ATCTAAGAATTTCACCTCTGACATCTG-3') for $P$. ovale. The amplified products were visualized in $1.5 \%$ agarose gels stained with ethidium bromide.

\section{Results}

The age distribution, number of people examined per category and percentage positive of the participants revealed that the highest number of infected patients were between 2130 years old (Table 1). Data obtained revealed that the highest incidence of malaria was amongst the adolescents; $47.4 \%$ from all 19 patients between ages 10 years and below, $32.5 \%$ equal distribution among all participating between ages 11-20 years and 21-30 years respectively. The older generations displayed lowest incidences of infection per head count (Table 1).
Table 1. Distribution of Plasmodium species in Relation to Age of Respondents.

\begin{tabular}{llll}
\hline Age/years & No. examined & Positive & Percentage (\%) \\
\hline $1-10$ & 19 & 9 & 47.4 \\
$11-20$ & 77 & 25 & 32.5 \\
$21-30$ & 118 & 38 & 32.2 \\
$31-40$ & 65 & 10 & 15.4 \\
$41-50$ & 28 & 3 & 10.7 \\
$51-60$ & 28 & 4 & 14.3 \\
61 and above & 15 & 4 & 13.3 \\
Total & 350 & 91 & 26.0 \\
\hline
\end{tabular}

$\mathrm{P}$ value $=0.043<0.05$.

Analysis of the DNA amplicons using Plasmodium genus genes revealed that 91 of the tested 350 specimens produced bands with a size of 240 base pairs, viewed with a 50 base pair ladder. A depiction of the first 19 specimens, some of which tested positive for the Plasmodium while others where negative is displayed (Figure 1). PCR analysis of the PCR products using Plasmodium falciparum gene specific primers revealed that all of the 91 specimens that tested positive using the Plasmodium genus specific primers formed bands of 206 base pairs, viewed with a 50 base pair ladder (Figure 2). The figure depicts 14 of the first Plasmodium falciparum positive specimens of the total 91 positives specimens analyzed. Analysis of the PCR products using Plasmodium malariae and P. ovale gene specific primers (rMAL1/rMAL2 and rOVA1/rOVA2) all did not produce any bands and where therefore tested negative (Figure 3).

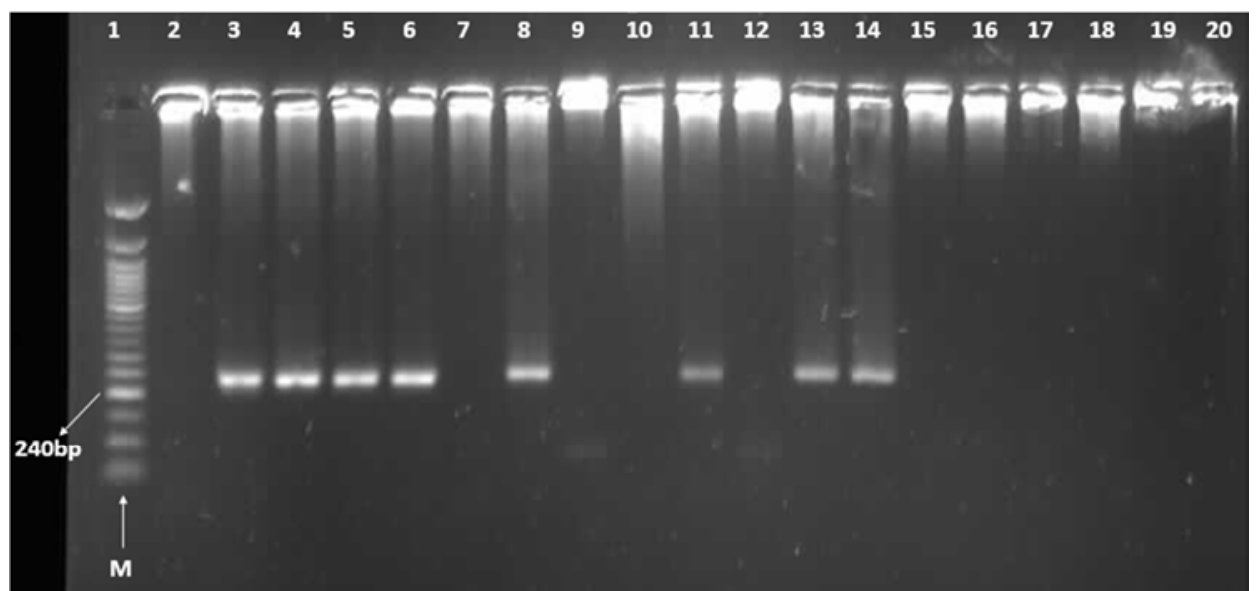

Figure 1. Agarose gel electrophoresis showing PCR products obtained for the first 20 specimens tested using genus specific primers: Lane 1 DNA marker, lanes 2-20 Plasmodium lane.

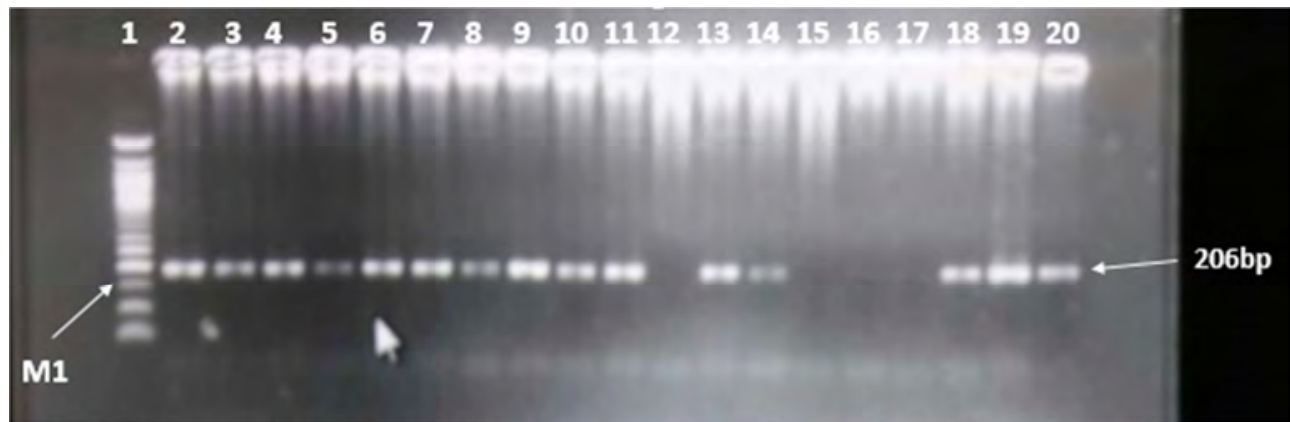

Figure 2. Agarose gel electrophoresis showing PCR products obtained using genus specific primers: Lane 1 DNA marker, lanes 2-19 Plasmodium falciparum, lane 20 Positive control. Lanes 2-11, 13-14, 18-19 all showed amplification and are positive for Plasmodium falciparum. 


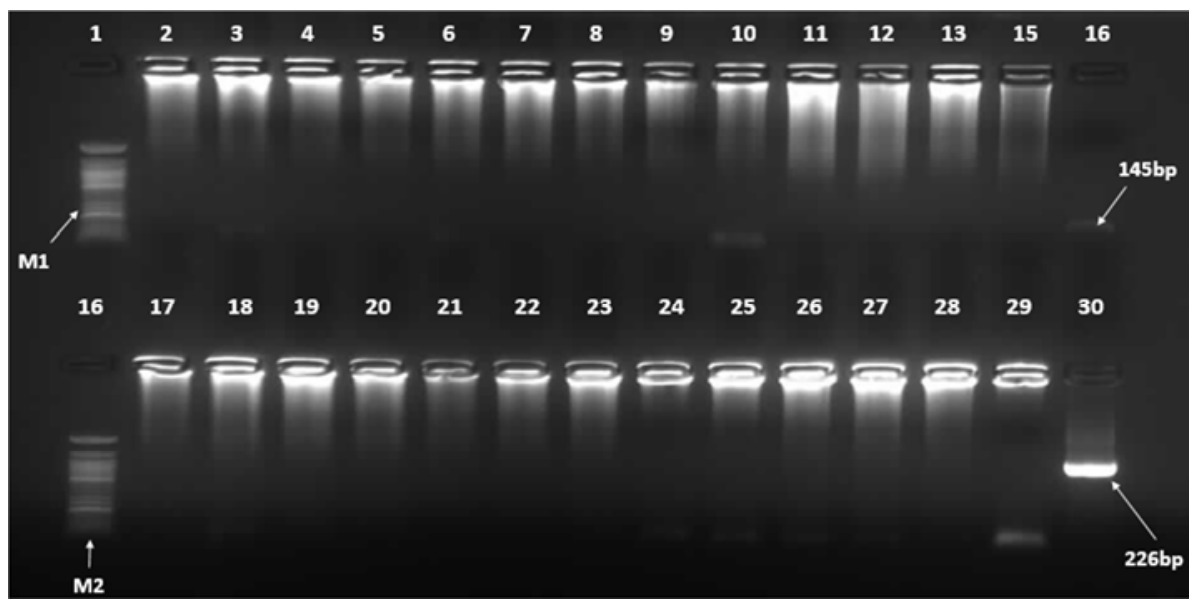

Figure 3. Agarose gel electrophoresis showing PCR products obtained using genus specific primers. Upper row Lanes 1 DNA marker (50bp), lanes 2-15 Plasmodium lane (Plasmodium malariae), Lane 16 positive control. Lower row Lane 16 DNA marker (50bp), lanes 17-29 Plasmodium lane (Plasmodium ovale), Lane 30 positive control. All test samples were negative.

In the present study, $62.86 \%$ (220) of the specimens were detected as Plasmodium-positive by microscopy (Table 2). On the other hand, of all the 350 patients, $70.86 \%$ (248) were determined as Plasmodium-positive using RDTs (Table 3). However, using both genus and specie-specific primers via nested PCR (nPCR) 91 (26\%) out of the total 350 patient samples were tested positive for malaria caused by Plasmodium falciparum (Tables 2 and 3).

\section{Discussion}

The prevalence of Plasmodium infections in northern Nigeria is high and widely distributed amongst all sexes' as well as socio-economic status in the country. Recent statistics from a neighboring state; Kaduna, revealed that the prevalence of malaria parasite amongst a similar number and distribution of participants stood at $35.7 \%$ [23]. In this study, the prevalence of the parasite was $26 \%$ (Table 1). Significant association was observed ( $p$ value $=0.043$ ) with respect to age with most of the percentage of the positive cases recorded were found within the underage children and elderly respondents that participated in this study. This could be attributed to the fact that the immune levels of under age categories are either low or underdeveloped [24]. This finding corroborates data obtained by Aduragbenro and colleagues [25] who reported that $25-30 \%$ of all mortalities caused by malaria in southern Nigeria was among children.

Data from this study showed that age groups 41-50 exhibited a substantial amount of resistance $(10.7 \%)$, so did age groups 61 and above (13.3\%), 51-60 (14.3\%) and age groups 31-40 (15.4\%).

These findings in this study suggest that the lower risk groups (31-40, 41-50, 61 and above) are less likely to be exposed to stagnant bodies of water; the ideal environment for mosquito propagation as could be the case for participants between ages 1-10 years who tested positive for malaria.

Furthermore, the data suggests that an acquired immunity may be also responsible for the gradually reducing incidence of infection (table 1).

Table 2. Comparison of microscopy and $n P C R$.

\begin{tabular}{lllllll}
\hline & Microscopy & & & & Total & \\
\hline & Positive & & Negative & & n & \% \\
\hline nPCR & N & \% & n & & & \\
Positive & & & & & & \\
Negative & 91 & 41.36 & 129 & 58.64 & 220 & 62.86 \\
Total & 0 & 0.0 & 130 & 100.0 & 130 & 37.14 \\
\hline
\end{tabular}

Table 3. Comparison of RDT and $n P C R$.

\begin{tabular}{|c|c|c|c|c|c|c|}
\hline & Rapid D & & & & & \\
\hline & Positive & & Negative & & Total & \\
\hline & $\mathbf{N}$ & $\%$ & $\mathrm{n}$ & $\%$ & $\mathbf{n}$ & $\%$ \\
\hline \multicolumn{7}{|l|}{ nPCR } \\
\hline Positive & 91 & 39.38 & 157 & 60.62 & 248 & 70.86 \\
\hline Negative & 0 & 0.0 & 102 & 100.0 & 102 & 29.14 \\
\hline Total & 91 & 26.0 & 259 & 74.0 & 350 & 100.0 \\
\hline
\end{tabular}


Asymptotic manifestation including chills, fever and sweating, nausea and general weakness presented by 259 participants in this study was due to some other infectious agent. Plasmodium analysis using the 4 respective genus specific primers revealed that only Plasmodium falciparum primers displayed amplification thus indicating that this specie alone was the most prevalent in the region, representing $26 \%$ (91 out of 350) of this study population (Figures 1-3). While such figures are within the reported statistics that revealed approximately a third of the populace from north central Nigeria are diagnosed with Plasmodium falciparum infection (36.6\%), particularly among children [23].

Data obtained in this study suggested that previously considered good standards for malaria detection via microscopic examination of blood smears by microscopy is inadequate for the diagnosis of malaria in this region (Table 2 ). The study showed that only $58.64 \%$ of the specimens identified as positive for malaria via microscopic technique were negative or false positives when compared against the nested PCR. This finding reaffirms the summation of other researchers which found that microscopic detection of Plasmodium infection is restricted to certain limits of parasitemia whereby if the latter falls below 100 parasites $/ \mathrm{ml}$, acute determination via microscopy becomes a problem [26]. This study also showed that while conventional microscopy displayed a specificity or ability to correctly detect patients who are actually not infected with malaria of $37.14 \%$, nested PCR revealed a specificity of $74 \%$ (Table 2 ).

The sensitivity and positive values of nPCR when microscopy was used as the reference standard was $100 \%$ and $41.36 \%$ respectively. In the same manner, the RDT kit utilized in this study revealed a $29.14 \%$ specificity value (Table 3). When RDTs were used as the reference standard, nPCR exhibited sensitivity and positive values of $100 \%$ and $39.38 \%$ respectively. This ability for PCR to more accurately detect low-grade parasitemia with a higher degree of sensitivity than microscopy and RDTs supports the reports by other researchers [22], [27-30]. The high false negative obtained using RDTs has been reported and suggested that all RDT negative results be confirmed by microscopic analysis [31].

A study showed that the critical success behind the identification of Plasmodium in the blood stems from the primers used which amplifies the dihydrofolate reductase (DHFR) and cytochrome oxidase III genes, peculiar to this genus [21], [32]. Precise detection should thus be achieved via recombinant DNA techniques.

The relatively low levels of Plasmodium falciparum infection via PCR (26\%) in comparison to that obtained by microscopy (62.86\%) and RDTs (70.86\%) may account for the dwindling potency of several over the counter antimalarial drugs in the country. It is conceivable that the misuse of anti-malarial drugs due to false positive results obtainable from conventional microscopic and RDT diagnosis maybe the culprit behind the emergence of drug resistant strains of Plasmodium. In the quest to combat
Plasmodium infections leading to the development of malaria, highly sensitive and specific techniques like PCR should become the prerequisite method for diagnosis, particularly in endemic areas like northern Nigeria.

\section{Conclusion}

This study underlined that nested PCR is a first-rate technique and a most have in all medical centers across Nigeria for the accurate determination of Plasmodium infection. Results obtained indicated also misdiagnosis associated with the microscopy (129 false positive), the previously considered gold standard and RDTS (157 false positive) is not allied to PCR based diagnosis (91 positive). The misuse of anti-malarial drugs that triggered the advent of drug resistant Plasmodium species in Nigeria can be attributed to the false positives recorded by microscopic and RDT analysis. Microscopic and RDT analysis of 350 blood specimens suggested that 220 and 248 samples respectively were infected with the Plasmodium pathogen. The most vulnerable group for malaria infection in the study area where children between ages 1-10 representing $47 \%$ of the tested patients within that age bracket. Regular screening via PCR is essential for the under-aged population for early detection and treatment of the specie specific Plasmodium pathogen in order to curb the rate of mortality across northern Nigeria. Discrepancies between PCR and microscopy results in diagnosis of malaria could be due to the reporting of artifacts by the latter as parasite giving false positive result, inadequate staining reagents and probably human error.

\section{References}

[1] Malik, A. M., Zaffar, N., Nadir, A., Malik, A. M., and Khan, R., (2010). Hematological Findings and Endemicity of Malaria in Gadap Region. Journal of the college of Physicians and Surgeons, 20:112-116.

[2] WHO: World Malaria Report 2011. Geneva: World Health Organization; 2011.

[3] WHO. World malaria report 2014. Geneva: World Health Organization; 2014.

[4] Tukur, A. I., (2010). Temporal Variation of Malaria occurrence in Kano Municipal Local Government Area. Bayero Journal of Pure and Applied Sciences, 3(1): 132-137.

[5] Das, L. K., and Pan, S. P., (2006) Clinical manifestation of severe form of $P$. falciparum malaria in Koraput district of Orissa state, India. Journal of Vector Borne Diseases, 43: 104-143.

[6] Alonso, P. L., Brown, G., Arevalo-Herrera, M., Binka, F., Chitnis, C., Collins, F., et al., (2011). A research agenda to underpin malaria eradication. PLos Medicine, 8.

[7] Lindblade, K. A., Steinhardt, L., Samuels, A., Kachur, S. P., and Slutsker, L., (2013). The silent threat: asymptomatic parasitemia and malaria transmission. Expert Review AntiInfective Therapy. 11: 623-639. 
[8] Zimmerman, P. A., Mehlotra, R. K., Kasehagen, L. J., and Kazura, J. W., (2004). Why do we need to know more about mixed Plasmodium species infections in humans? Trends in Parasitology, 20: 440-447.

[9] Muller, I., Zimmerman, P. A., and Reeder, J. C., (2007): Plasmodium malariae and Plasmodium ovale - the 'bashful' malaria parasites. Trends in Parasitology, 23(6): 278-283.

[10] Mayxay, M., Pukrittayakamee, S., Newton, P. N., and White, N. J., (2004). Mixed-species malaria infections in humans. Trends in Parasitology, 20: 233-240.

[11] Moody, A., (2002). Rapid diagnostic tests for malaria parasites. Clinical Microbiology Reviews, 15: 66-78.

[12] Ndao, M., Bandyayera, E., Kokoskin, E., Gyorkos, T. W., MacLean, J. D., and Ward, B. J., (2004). Comparison of blood smear, antigen detection, and nested-PCR methods for screening refugees from regions where malaria is endemic after a malaria outbreak in Quebec, Canada. Journal of Clinical Microbiology, 42: 2694-2700.

[13] Bell, D. R., Wilson, D. W., and Martin, L. B., (2005). Falsepositive results of a Plasmodium falciparum histidine-rich protein 2-detecting malaria rapid diagnostic test due to high sensitivity in a community with fluctuating low parasite density. American Journal of Tropical Medicine and Hygiene, 73: 199-203.

[14] Murray, C. K., Gasser, R. A. Jr., Magill, A. J., and Miller, R. S., (2008). Update on rapid diagnostic testing for malaria. Clinical Microbiology Reviews, 21: 97-110.

[15] Coleman, R. E., Sattabongkot, J., Promstaporm, S., Maneechai, N., Tippayachai, B., Kengluecha, A., Rachapaew, N., Zollner, G., Miller, R. S., Vaughan, J. A., Thimasarn, K., and Khuntirat, B., (2006). Comparison of PCR and microscopy for the detection of asymptomatic malaria in a Plasmodium falciparum/vivax endemic area in Thailand. Malaria Journal, 14: 121.

[16] Okell, L. C., Ghani, A. C., Lyons, E., and Drakeley, C. J., (2009). Submicroscopic infection in Plasmodium falciparumendemic populations: a systematic review and meta-analysis. Journal of Infectious Diseases, 200: 1509-1517.

[17] Cheng, Q., Cunningham, J., and Gatton, M. L., (2015). Systematic Review of Sub-microscopic P. vivax infections: prevalence and determining factors. PLos Neglected Tropical Diseases, 9.

[18] Perandin, F., Manca, N., Calderaro, A., Piccolo, G., Galati, L., Ricci, L., Medici, M. C., Arcangeletti, M. C., Snounou, G., Dettori, G., and Chezzi, C., (2004). Development of a realtime PCR assay for detection of Plasmodium falciparum, Plasmodium vivax, and Plasmodium ovale for routine clinical diagnosis. Journal of Clinical Microbiology, 42: 1214-1219.

[19] Rougemont, M., Van Saanen, M., Sahli, R., Hinrikson, H. P., Bille, J., and Jaton, K., (2004). Detection of four Plasmodium species in blood from humans by $18 \mathrm{~S}$ rRNA gene subunitbased and species-specific real-time PCR assays. Journal of Clinical Microbiology, 42: 5636-5643.

[20] Taylor, B. J., Martin, K. A., Arango, E., Agudelo, O. M., Maestre, A., and Yanow, S. K., (2011). Real-time PCR detection of Plasmodium directly from whole blood and filter paper samples. Malaria Journal, 10: 244.

[21] Silbermayr, K., Eigner, B., Duscher, G. G., Joachim, A., and Fuehrer, H. P., (2014). The detection of different Dirofilaria species using direct PCR technique. Parasitology Research Journal, 113: 513-516.

[22] Snounou, G., and Singh, B., (2002). Nested PCR analysis of Plasmodium parasites. Methods in Molecular Medicine, 72: 189-203.

[23] Umaru, M. L., and Uyaiabasi, G. N. (2015). Prevalence of Malaria in Patients Attending the General Hospital Makarfi, Makarfi Kaduna-State, North-Western Nigeria. American Journal of Infectious Diseases and Microbiology, 3(1): 1-5.

[24] Paniker, C. K. J., (2007). Medical Parasitology. (6th edn.). Jaypee Brothers Medical Publishers (P) Ltd, New Delhi, India. pp 65-95.

[25] Aduragbenro, D. A., Falade, C. O., Kotila, R. T., and Ademowo, G. O., (2007). Age as a risk factor for thrombocytopenia and anemia in children treated for acute uncomplicated falciparum malaria. Journal of Vector Borne Diseases, 44: 266-271.

[26] Joanny, F., Lohr, S. J., Engleitner, T., Lell, B., and Mordmuller, B., (2014). Limit of blank and limit of detection of Plasmodium falciparum thick blood smear microscopy in a routine setting in Central Africa. Malaria Journal, 13: 234.

[27] Johnston, S. P., Pieniazek, N. J., Xayavong, M. V., Slemenda, S. B., Wilkins, P. P., and da Silva, A. J., (2006). PCR as a confirmatory technique for laboratory diagnosis of malaria. Journal of Clinical Microbiology, 44: 1087-1089.

[28] Buppan, P., Putaporntip, C., Pattanawong, U., Seethamchai, S., and Jongwutiwes, S., (2010). Comparative detection of Plasmodium vivax and Plasmodium falciparum DNA in saliva and urine samples from symptomatic malaria patients in a low endemic area. Malaria Journal, 9: 72.

[29] Osman, M. M., Nour, B. Y., Sedig, M. F., De Bes L., Babikir, A. M., Mohamedani, A. A., and Mens, P. F., (2010). Informed decision-making before changing to RDT: a comparison of microscopy, rapid diagnostic test and molecular techniques for the diagnosis and identification of malaria parasites in Kassala, eastern Sudan. Tropical Medicine and International Health, 15: 1442-1448.

[30] Lima, G. F., Levi, J. E., and Geraldi, M. P., (2011). Malaria diagnosis from pooled blood Samples; Comparative analysis of real-time PCR, nested PCR and Immunoassay as a platform for the molecular and serological diagnosis of malaria on a largescale. Memorias do Instituto Oswaldo Cruz, 106(6): 691-700.

[31] Leke, R. F., (2009). Detection of the Plasmodium falciparum antigen HRP - 2 in blood of pregnant women: implication for diagnosing placental malaria. Journal of Clinical Microbiology 37: 2992-2996.

[32] Echeverry, D. F., Deason, N. A., Davidson, J., Makuru, V., Xiao, H., Niedbalski, J., Kern, M., Russell, T. L., Burkot, T. R., Collins, F. H., and Lobo, N. F., (2016). Malaria Journal, 15: $1-12$. 